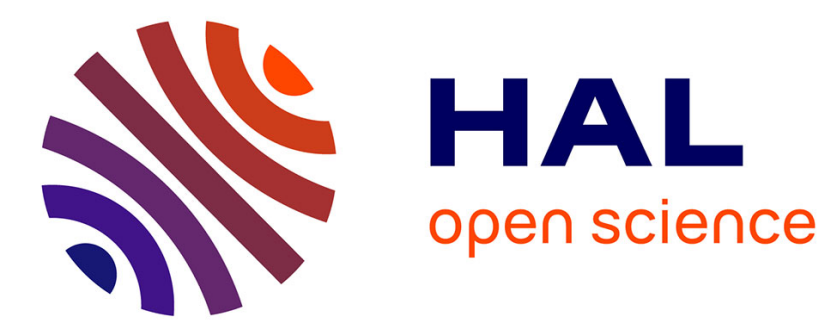

\title{
Spectre des fluctuations thermiques de la surface libre d'un liquide simple
}

\author{
M. A. Bouchiat, J. Meunier
}

\section{To cite this version:}

M. A. Bouchiat, J. Meunier. Spectre des fluctuations thermiques de la surface libre d'un liquide simple. Journal de Physique, 1971, 32 (7), pp.561-571. 10.1051/jphys:01971003207056100 . jpa-00207111

\section{HAL Id: jpa-00207111 https://hal.science/jpa-00207111}

Submitted on 1 Jan 1971

HAL is a multi-disciplinary open access archive for the deposit and dissemination of scientific research documents, whether they are published or not. The documents may come from teaching and research institutions in France or abroad, or from public or private research centers.
L'archive ouverte pluridisciplinaire HAL, est destinée au dépôt et à la diffusion de documents scientifiques de niveau recherche, publiés ou non, émanant des établissements d'enseignement et de recherche français ou étrangers, des laboratoires publics ou privés. 
Classification

Physics Abstracts :

$14.82,14.87$

\title{
SPECTRE DES FLUCTUATIONS THERMIQUES DE LA SURFACE LIBRE D'UN LIQUIDE SIMPLE
}

\author{
M. A. BOUCHIAT et J. MEUNIER \\ Laboratoire de Spectroscopie Hertzienne de l'E. N. S., associé au C. N. R. S. \\ Faculté des Sciences de Paris (France)
}

(Reçu le 5 mars 1971)

\begin{abstract}
Résumé. - Le spectre des fluctuations thermiques de la coordonnée verticale des points de la surface libre d'un liquide est calculé en recherchant la solution des équations linéarisées de l'hydrodynamique satisfaisant à des conditions initiales particulières connues, choisies d'après la description statistique du système à l'équilibre thermique. On montre que, pour obtenir la solution rigoureuse du problème, il ne faut pas considérer seulement l'état statistique de la surface, mais aussi celui du fluide sous-jacent. On calcule par ailleurs la fonction de réponse de la surface à une force de pression extérieure de caractère sinusoïdal et on vérifie que le spectre obtenu satisfait au théorème de fluctuation-dissipation. Dans certaines conditions d'amortissement voisines de l'amortissement critique, il présente des différences non négligeables vis-à-vis du spectre des fluctuations thermiques d'un oscillateur harmonique. Ces différences sont liées aux phénomènes de retard et de dissipation, qui accompagnent la propagation du mouvement de la surface, aux couches fluides voisines. On montre sur un spectre expérimental que l'effet prévu est observable.
\end{abstract}

\begin{abstract}
The power spectrum of fluctuations thermally excited on the free surface of a simple liquid is calculated by looking for a solution of the linearized hydrodynamic equations which satisfy given initial conditions, specified by considerations of equilibrium statistical mechanics. To obtain the exact solution it is necessary to consider the equilibrium properties, not only of the liquid surface, but also of the fluid underneath. Furthermore we compute the response function of the surface to the action of an external sinusoidal pressure and verify the fluctuation-dissipation theorem. In certain damping conditions (near the critical damping), the power spectrum exhibits non-negligible deviations from the power spectrum of the displacement, for a thermally excited harmonic system. These differences are connected with the time delay with which a motion at the surface is felt underneath, and with the corresponding dissipation process. We show an experimental spectrum which is in good agreement with the predicted line shape.
\end{abstract}

$\mathrm{Du}$ fait de l'agitation thermique, la surface libre d'un liquide n'est pas parfaitement plane. Les aspérités excitées thermiquement ont été étudiées par diffusion de la lumière dès les années 1913-1925 par Mandelshtam, puis Raman et Ramdas [1]. En fixant l'angle d'observation par rapport à la direction de réflexion régulière, on détermine le vecteur d'onde des fluctuations observées. A présent, grâce à la monochromaticité et la directivité d'un laser, il est devenu aussi possible d'étudier l'évolution temporelle des fluctuations [2]. Des arguments d'ordre qualitatif permettent de prévoir l'allure générale du spectre, et dès les premières expériences, on a pu observer l'aspect attendu: doublet pour un liquide très peu visqueux à la surface duquel les ondes se propagent en s'amortissant lentement, raie unique centrée à la fréquence de la lumière incidente pour un liquide très visqueux sur lequel les ondes s'amortissent, sans se propager. Une expérience de diffusion de la lumière peut maintenant être utilisée pour mesurer certains paramètres du liquide (tension superficielle, viscosités) sans perturber en principe son état d'équilibre.
Le problème qui nous intéresse ici est celui de la relation qui lie ces paramètres à la forme spectrale de la lumière diffusée. Dans les 2 cas extrêmes que nous venons de mentionner, l'analyse du spectre formé de composantes lorentziennes est extrêmement simple, il n'en est plus du tout de même dans les cas d'amortissement intermédiaires, en particulier à l'approche du point d'amortissement critique (transition du régime apériodique au régime périodique amorti). Or, un certain nombre de problèmes physiques intéressants correspondent précisément à des situations de ce type (étude de l'interface liquide-gaz d'un fluide pur au voisinage du point critique, étude de la surface libre d'un liquide nématique). Nous nous limiterons ici au cas de la surface libre d'un liquide simple: le calcul s'adapte facilement aux autres cas où la loi de dispersion des ondes est seulement plus complexe.

Le problème théorique du spectre des fluctuations de la coordonnée verticale des points d'une surface libre peut être traité à l'aide du procédé suggéré par Landau et Placzek [3] et appliqué par Mountain [4] à l'étude des fluctuations de densité en volume: on 
recherche la solution des équations de l'hydrodynamique satisfaisant à des conditions initiales particulières connues et l'on déduit ces conditions initiales de la description statistique du système à l'équilibre thermique. Le problème avait déjà été abordé de la sorte dans une précédente publication [5]. On avait pris en considération l'état statistique de la surface libre à l'équilibre thermique et on avait déduit l'évolution temporelle des fluctuations d'une solution particulière, de type exponentiel, du problème hydrodynamique des ondes de surface. Physiquement, le procédé utilisé revenait à calculer les fluctuations thermiques de la surface en l'assimilant à une membrane élastique infiniment mince ayant les mêmes modes de vibration qu'elle, ou encore, à traiter l'excitation thermique des ondes de capillarité comme celle d'un gaz libre de phonons de surface. On obtenait ainsi un spectre identique à celui des fluctuations de la coordonnée d'un oscillateur harmonique excité thermiquement.

Dans ce travail, nous commençons (partie A) par rappeler le calcul précédent, puis nous faisons remarquer que la solution adoptée revient à faire implicitement une certaine hypothèse sur l'état statistique du fluide en volume. Or il existe des cas où cette hypothèse n'est pas raisonnable. Ainsi par exemple, on serait conduit à supposer qu'il existe une certaine corrélation entre les valeurs au même instant du déplacement de la surface et de la composante verticale de la vitesse du fluide en profondeur (l'importance de la corrélation à admettre étant surtout grande dans les conditions d'amortissement voisines des conditions critiques). Ceci nous a conduit à reformuler le problème, dans le présent travail, en prenant en considération à la fois l'état statistique de la surface libre et celui du fluide sous-jacent (partie B). Nous obtenons le spectre des fluctuations en résolvant le problème hydrodynamique de manière beaucoup plus générale et rigoureuse que précédemment et en imposant que le déplacement à la surface et la vitesse en profondeur au même instant, soient des variables statistiquement indépendantes. Nous calculons par ailleurs la fonction de réponse de la surface à une force de pression extérieure de caractère sinusoïdal. A l'aide de ce dernier résultat, nous pouvons vérifier que le nouveau spectre obtenu, qui diffère du précédent, satisfait bien au théorème de fluctuation-dissipation. Dans une dernière partie $(C)$, nous comparons numériquement le spectre obtenu dans ce travail pour les fluctuations de surface à celui des fluctuations thermiques d'un oscillateur harmonique. Dans certaines conditions d'amortissement voisines de l'amortissement critique, l'écart entre les deux types de spectre n'est pas négligeable. On montre un spectre expérimental sur lequel l'effet prévu est observable.

A) Rappels sur des résultats antérieurs. - 1) DÉFINITIONS ET PROPRIÉTÉS CARACTÉRISTIQUES DE LA FONCTION DE CORRÉLATION. - Nous nous intéressons au déplacement vertical d'un petit élément de surface dont les dimensions sont choisies à la fois très grandes devant les dimensions moléculaires et très petites devant la distance caractéristique de la périodicité des fluctuations étudiées. Soit $\zeta(\mathbf{r}, t)$ le déplacement vertical au temps $t$ de l'élément de la surface dont la projection sur le plan horizontal est définie par $\mathbf{r}$. Pour un liquide à l'équilibre, la variable macroscopique $\zeta(\mathbf{r}, t)$ est aléatoire ; on la définit de telle sorte que $\overline{\zeta(\mathbf{r}, t)}=0$. Pour construire la fonction de corrélation spatio-temporelle $\overline{\zeta(\mathbf{r}, t) \zeta(\mathbf{r}+\boldsymbol{\rho}, t+\tau)}$, nous tiendrons compte des propriétés suivantes: 1) le processus aléatoire est stationnaire ; 2) les propriétés de la surface sont invariantes par translation. La fonction de corrélation ne dépend donc que des variables $\tau$ et $\boldsymbol{\rho}$, elle sera désignée par $g(\boldsymbol{\rho}, \tau)$. Il est utile de décomposer $\zeta(\mathbf{r}, t)$ en modes spatiaux de vecteur d'onde q :

$$
\zeta_{\mathbf{q}}(t)=A^{-1} \int \zeta(\mathbf{r}, t) \exp (\mathbf{i q} \cdot \mathbf{r}) \mathrm{d}^{2} r
$$

(At, aire de la surface liquide). L'invariance de la surface par translation entraîne :

$$
\begin{aligned}
\overline{\zeta_{\mathbf{q}^{\prime}}(t) \zeta_{\mathbf{q}}^{*}(t+\tau)}=\mathcal{A}^{-2} \int & \mathrm{d}^{2} \rho g(\rho, \tau) \exp \frac{\mathrm{i}\left(\mathbf{q}+\mathbf{q}^{\prime}\right) \cdot \boldsymbol{\rho}}{2} \times \\
& \times \int \mathrm{d}^{2} R \operatorname{exp~i}\left(\mathbf{q}^{\prime}-\mathbf{q}\right) \cdot \mathbf{R}
\end{aligned}
$$

avec

$$
\mathbf{R}=\frac{\mathbf{r}^{\prime}+\mathbf{r}}{2} \quad \text { et } \quad \boldsymbol{\rho}=\mathbf{r}^{\prime}-\mathbf{r}
$$

Le second membre est nul sauf si $\mathbf{q}=\mathbf{q}^{\prime}$ et $g(\boldsymbol{\rho}, \tau)$ se décompose en une somme de fonctions $\left({ }^{1}\right)$ de corrélation $G_{\mathbf{q}}(\tau)$ relatives à chacun des modes $\mathbf{q}$ :

$$
\begin{aligned}
g(\boldsymbol{\rho}, \tau) & =\mathcal{A}(2 \pi)^{-2} \int \mathrm{d}^{2} q \overline{\zeta_{\mathbf{q}}(t) \zeta_{\mathbf{q}}^{*}(t+\tau)} \mathrm{e}^{\mathrm{i} \mathbf{q} \cdot \boldsymbol{\rho}} \\
& =(2 \pi)^{-2} \int \mathrm{d}^{2} q G_{\mathbf{q}}(\tau) \mathrm{e}^{\mathrm{i} \mathbf{q} \cdot \boldsymbol{\rho}}
\end{aligned}
$$

L'expérience permet d'atteindre soit la fonction de corrélation du champ électrique diffusé par les fluctuations de vecteur d'onde $\mathbf{q}$ fixé, proportionnelle à $G_{\mathbf{q}}(\tau)$, soit sa transformée de Fourier :

$$
P_{\mathbf{q}}(\omega)=(2 \pi)^{-1} \int_{-\infty}^{+\infty} \mathrm{d} \tau G_{\mathbf{q}}(\tau) \mathrm{e}^{\mathrm{i} \omega \tau} .
$$

2) Modèle STATistiQue. - Indiquons de manière générale la méthode utilisée pour construire la fonction de corrélation $\overline{X(0) X(\tau)}$ d'une variable d'état $X$; nous l'appliquerons ensuite à $X=\zeta_{\mathbf{q}}$.

(1) Pour un liquide ordinaire, les propriétés de la surface sont aussi invariantes par rotation, $g(\rho, \tau)$ ne dépend que de $|\boldsymbol{\rho}|$ et $G_{\mathbf{q}}(\tau)$ ne dépend que de $|\mathbf{q}|$. 
Désignons par $\left\{x_{k}\right\}$ un ensemble complet de variables d'état pour le type de système considéré $\left({ }^{2}\right)$. Par exemple, pour le problème qui nous intéresse, il figurera parmi les $x_{k}$, $\zeta_{\mathbf{q}}$ et $\dot{\zeta}_{\mathbf{q}}$.

Considérons un ensemble de systèmes thermodynamiques identiques; pour chacun de ces systèmes $X(t), x_{k}(t)$, varient autour de la valeur d'équilibre, supposée nulle. A un instant donné $t=0$, on peut séparer cet ensemble en différentes classes, avec la convention suivante: tous les systèmes d'une même classe sont dans un même état initial (défini par les valeurs $\left\{x_{k}(0)\right\}$ ), les systèmes de deux classes distinctes sont dans des états initiaux différents.

Intéressons-nous d'abord à l'évolution de $X$ pour les systèmes d'une même classe. Nous admettrons que l'évolution moyenne (c'est-à-dire la valeur moyenne de $X(t)$ prise sur les systèmes d'une même classe) obéit à un système d'équations macroscopiques adapté à la description du fluide (hypothèse d'Onsager), ici les équations de l'hydrodynamique $\left({ }^{3}\right)$. Comme les écarts par rapport à la position d'équilibre sont toujours petits, nous pouvons linéariser ces équations. Ceci permet de prévoir que, de manière très générale, $X(t)$ sera à la fois fonction de $t$ et fonction linéaire des $x_{k}(0)$ :

$$
X(t)=\sum_{k} x_{k}(0) f_{k}(t), \quad t>0 .
$$

Nous obtenons ainsi pour les systèmes d'une classe la valeur moyenne de l'expression à calculer :

$$
X(0) X(t)=\sum_{k} X(0) x_{k}(0) f_{k}(t), \quad t>0 .
$$

Envisageons maintenant les différentes classes. De l'une à l'autre, $x_{k}(0)$ varie c'e manière aléatoire, mais $f_{k}(t)$ ne change pas. L'expression ci-dessus corsidérée pour des systèmes appartenant à des classes cifférentes se comporte comme une fonction certaine çu temps et une fonction aléatoire de $x_{k}(0)$. Effectuant la moyenne sur toutes les classes, nous obtenors la forction de corrélation :

$$
\overline{X(0) X(t)}=\sum_{k} \overline{X(0) x_{k}(0)} f_{k}(t), \quad t>0 .
$$

Etant donné la manière dont elle a été construite, cette expression n'est valable que pour $t>0$. On peut obtenir sa valeur pour $t$ quelconque en remarquant simplement que c'est une fonction paire de $t$ du fait de la stationnarité du processus aléatoire :

$$
\overline{X(0) X(t)}=\sum_{k} \overline{X(0) x_{k}(0)} f_{k}(|t|) .
$$

(2) $\mathrm{Si}$ à $t=0$ le système s'écarte de manière macroscopique de l'état d'équilibre, la donnée à cet instant des va'eurs prises par toutes les variables $x_{k}$ suffit à spécifier comp'ètement à tout instant ultérieur l'état du système régi par les lois de l'hydrodynamique. Les valeurs prises par l'indice $k$ peuvent être discrètes ou continues.

(3) Ce procédé a été suggéré par Landau et Placzek [3] et utilisé par Mountain [4] pour l'étude des fluctuations de densité en volume.
Les valeurs moyennes qui figurent dans le second membre de l'équation (8) s'évaluent en appliquant les principes de la mécanique statistique à un système àl'équilibre.

En résumé, le problème du calcul du spectre des fluctuations se ramène, d'une part, à la recherche de la solution des équations de l'hydrodynamique satisfaisant à des conditions initiales particulières connues et, d'autre part, au choix de ces conditions initiales d'après la description statistique du système à l'équilibre thermique.

L'examen de l'équation (8) suscite une remarque très utile pour la suite : $\mathrm{Si}$ une ou plusieurs variables d'état $x_{1} \ldots x_{j}$ sont statistiquement indépendantes de $X$ (c'est-à-dire $\left.\overline{X(0) x_{j}(0)}=0\right)$, on peut construire la fonction de corrélation de $X$ directement à partir de la solution du problème hydrodynamique correspondant aux conditions initiales $x_{1}(0)=0 \ldots x_{j}(0)=0$.

3) RAPPEL SUR LE PROBLÈME HYDRODYNAMIQUE ET UNE MÉTHODE DE RÉSOLUTION [6]. - On s'intéresse à des déplacements transversaux du liquide (perpendiculaires à q) qui ne sont possibles (dans un milieu dont le coefficient élastique de rigidité est considéré comme nul) qu'au voisinage immédiat de la surface de séparation avec un autre fluide. La force de rappel due essentiellement aux forces de capillarité $\left({ }^{4}\right)$ est très faible et le mouvement s'effectue à des fréquences basses pour lesquelles le fluice peut être considéré comme incompressible.

Les équations de l'hydrodynamique linéarisées, traduisant la conservation de la masse et de l'impulsion dans le volume du liquide, sont utilisées sous la forme :

$$
\begin{gathered}
\operatorname{div} \mathbf{v}=0 \\
\frac{\partial \mathbf{v}}{\partial t}=-\frac{1}{\rho} \operatorname{grad} \mathrm{p}+\frac{\eta}{\rho} \Delta \mathbf{v}
\end{gathered}
$$

( $\rho$ densité, $\eta$ viscosité, $\mathbf{v}$ vitesse, $\mathbf{p}$ pression du fluide).

De plus, le mouvement doit satisfaire aux conditions aux limites qui expriment que les contraintes normales et targentielles s'exerçant sur un petit élément de la surface ce séparation sont les mêmes de part et d'autre :

$$
\left.\begin{array}{l}
-p+2 \eta \frac{\partial v_{z}}{\partial z}-\alpha \frac{\partial^{2} \zeta}{\partial x^{2}}=-p_{0} \\
\eta\left(\frac{\partial v_{x}}{\partial z}+\frac{\partial v_{z}}{\partial x}\right)=0
\end{array}\right\} \grave{a ̀ ~ l a ~ s u r f a c e ~ l i b r e ~}
$$

( $\alpha$ tension superficielle, $p_{0}$ pression dans la phase gazeuse au-dessus du liquide). On a choisi l'axe $O x$ cirigé suivant $\mathbf{q}$ et l'axe $O z$ vertical, le liquide étant citué dans la région $z<0$.

(4) Nous négligeons ici l'effet des forces de gravité qui interviendrait à des longueurs d'onde plus grandes. 
L'épaisseur du liquide est supposée très grande devant $q^{-1}$ et le fond du récipient empêche tout mouvement en profondeur

$$
\mathbf{v} \rightarrow 0 \quad \text { lorsque } \quad z \rightarrow-\infty
$$

L'amplitude du déplacement étant petite devant la longueur d'onde, on peut faire l'approximation

$$
v_{z}=\frac{\mathrm{d} \zeta}{\mathrm{d} t}=\frac{\partial \zeta}{\partial x} \frac{\mathrm{d} x}{\mathrm{~d} t}+\frac{\partial \zeta}{\partial t} \approx \frac{\partial \zeta}{\partial t}
$$

à la surface.

On introduit les paramètres :

$$
\begin{aligned}
\tau_{0} & =\frac{\rho}{2 \eta q^{2}} \\
y & =\frac{\alpha \rho}{4 \eta^{2} q}
\end{aligned}
$$

caractéristiques du fluide étudié. Dans toute la suite, $q$ désigne $|\mathbf{q}|(q>0)$.

Levich [6] a recherché des solutions particulières des équations de type exponentiel, c'est-à-dire proportionnelles à $\mathrm{e}^{\mathrm{i} q x} \mathrm{e}^{\alpha t} \mathrm{e}^{m z}$. Il a obtenu ainsi pour solutions la partie réelle des fonctions suivantes :

$$
\begin{aligned}
& v_{x}=\sum_{v=1,2}-\mathrm{ie}^{-\mathrm{i} q x+S_{v} t / \tau_{0}}\left(A_{v} \mathrm{e}^{q z}+B_{v} \mathrm{e}^{m_{v} z}\right) \\
& v_{z}=\sum_{v=1,2} \mathrm{e}^{-\mathrm{i} q x+S_{v} t / \tau_{0}}\left(A_{v} \mathrm{e}^{q z}+\frac{q}{m_{v}} B_{v} \mathrm{e}^{m_{v} z}\right) \\
& \zeta=\sum_{v=1,2} \mathrm{e}^{-\mathrm{i} q x+S_{v} t / \tau_{0}} \cdot \frac{\tau_{0}}{S_{v}}\left(A_{v}+\frac{q}{m_{v}} B_{v}\right) \\
& \mathrm{p}=\mathrm{p}_{0}-\sum_{v} \frac{S_{v} \rho}{q \tau_{0}} A_{v} \mathrm{e}^{-\mathrm{i} q x+S_{v} \tau / \tau_{0}+q z}
\end{aligned}
$$

$\operatorname{avec}\left({ }^{5}\right)$

$$
\begin{aligned}
& m_{v}=q \sqrt[+]{1+2} \overline{S_{v}} \\
& \frac{A_{v}}{B_{v}}=-\frac{m_{v}^{2}+q^{2}}{2 q m_{v}}
\end{aligned}
$$

et $S_{v}$ devant satisfaire simultanément à l'équation de dispersion :

$$
y+(S+1)^{2}-\sqrt[+]{1+2 S}=0
$$

et à la condition

$$
\operatorname{Re} S<0 .
$$

Pour chaque valeur de $y$, il existe 2 valeurs $S_{1}$ et $S_{2}$ satisfaisant les 2 relations (15). Ces valeurs sont réelles pour $y<0,145$ (système apériodique) et imaginaires conjuguées pour $y>0,145$ (système périodique amorti).

(5) On désigne par $\sqrt[+]{ }$ la détermination de la racine à partie réelle positive.
La solution ci-dessus fait intervenir 2 constantes arbitraires (par exemple $A_{1}$ et $A_{2}$ ). On peut vérifier que celles-ci sont complètement déterminées si l'on choisit de décrire les conditions initiales à l'aide de $\zeta_{q}(0)$ et $\dot{\zeta}_{q}(0)$ :

$$
\begin{gathered}
A_{v}=\frac{\zeta_{\mathbf{q}}(0) S_{\mu}-\dot{\zeta}_{\mathbf{q}}(0)}{\left(S_{\mu}-S_{v}\right) \tau_{0}} \frac{\tau_{0}}{\left(1+S_{v}\right)} \\
B_{v}=-\frac{\zeta_{\mathbf{q}}(0) S_{\mu}-\dot{\zeta}_{\mathbf{q}}(0) \tau_{0}}{\left(S_{\mu}-S_{v}\right) \tau_{0}}+\sqrt{1+2 S_{v}^{-}}
\end{gathered}
$$

(les indices $v$ et $\mu$ correspondant à 1 ou 2 avec la convention $v \neq \mu$ ).

4) CALCUl DiRect De LA FONCTION DE CORRÉLATION DES FLUCTUATIONS THERMIQUES DE LA SURFACE D'UN LIQUIDE [5]. - Puisque nous disposons d'une solution (expressions 12) du problème hydrodynamique, nous pouvons tout de suite penser l'utiliser pour construire la fonction de corrélation de $\zeta_{q}(t)$. Cette solution est complètement spécifiée par la donnée de $\zeta(\mathbf{r}, 0)$ et $\dot{\zeta}(\mathbf{r}, 0)$ ou de $\zeta_{\mathbf{q}}(0)$ et $\dot{\zeta}_{\mathbf{q}}(0)$. Il nous reste donc à évaluer $\overline{\left|\zeta_{\mathbf{q}}(0)\right|^{2}}$ et $\overline{\zeta_{\mathbf{q}}(0) \dot{\zeta}_{\mathbf{q}}(0)}$ à l'équilibre thermique :

$$
\begin{gathered}
\overline{\left|\zeta_{\mathbf{q}}(0)\right|^{2}}=\frac{k_{B} T}{\alpha q^{2} \mathcal{A}} \\
\overline{\zeta_{\mathbf{q}}(0) \dot{\zeta}_{\mathbf{q}}(0)}=0 .
\end{gathered}
$$

D'après ce qui précède, nous pouvons déduire $G_{\mathbf{q}}(\tau)$ de l'expression $(12 c)$ calculée pour $\dot{\zeta}_{\mathbf{q}}(0)=0$ et $\zeta_{\mathbf{q}}(0)$ arbitraire :

$$
\zeta_{\mathbf{q}}(t)=\zeta_{\mathbf{q}}(0)\left(S_{2}-S_{1}\right)^{-1}\left(S_{2} \mathrm{e}^{S_{1} t / \tau_{0}}-S_{1} \mathrm{e}^{S_{2} t / \tau_{0}}\right) .
$$

Nous obtenons :

$$
G_{\mathbf{q}}(\tau)=\frac{k_{B} T}{\alpha q^{2}}\left(S_{2}-S_{1}\right)^{-1}\left[S_{2} \mathrm{e}^{S_{1}\left|\tau / \tau_{0}\right|}-S_{1} \mathrm{e}^{S_{2}\left|\tau / \tau_{0}\right|}\right]
$$

$$
P_{\mathbf{q}}(\omega)=\frac{k_{B} T}{\pi \alpha q^{2}} \frac{S_{2} S_{1}\left(S_{2}+S_{1}\right) \tau_{0}}{\left(\omega^{2} \tau_{0}^{2}-S_{1} S_{2}\right)^{2}+\omega^{2} \tau_{0}^{2}\left(S_{1}+S_{2}\right)^{2}}
$$

On rappelle que $S_{1}$ et $S_{2}$ sont les 2 valeurs de $S$ qui vérifient simultanément les 2 relations (15).

B) Reformulation du problème. - 1) CRITIQUe RELATIVE AU CALCUL DE LA FONCTION DE CORRÉLATION DE $\zeta_{\mathbf{q}}$ - Nous venons de rappeler un calcul

(6) Le carré moyen d'une fluctuation thermodynamique se calcule en évaluant le travail minimum $R_{\min }$ nécessaire pour faire apparaître une fluctuation, ici $\frac{1}{2} \alpha q^{2} \mathcal{A} \zeta \mathbf{q}^{2}$.

(7) Cette propriété est valable de manière tout à fait générale pour une variable d'état d'un système physique obéissant aux lois de la mécanique classique. 
simple de la fonction de corrélation de $\zeta_{\mathbf{q}}$. Il utilise une solution mathématique particulière du problème hydrodynamique qui est définie par la donnée de $\zeta_{q}(0)$ et $\dot{\zeta}_{q}(0)$, c'est-à-dire de paramètres relatifs à la surface seule. La fonction de corrélation des fluctuations a été ainsi construite en faisant intervenir l'état statistique de la surface, sans s'intéresser à celui du fluide sous-jacent. Physiquement, ce procédé revient à assimiler la surface liquide à une membrane élastique ayant les mêmes modes de vibration. Dans ces conditions, le spectre obtenu est identique au spectre des fluctuations de la coordonnée d'un oscillateur harmonique excité thermiquement.

En fait, si on s'interroge sur l'état statistique du fluide en volume, on est conduit à faire une remarque importante. La solution mathématique que nous avons utilisée, fournit pour $v_{z}$ l'expression $(12 b)$. Si l'on explicite $A_{v}$ et $B_{v}$ en fonction de $\zeta_{q}(0)$ et $\dot{\zeta}_{\mathbf{q}}(0)$ et si l'on introduit la transformée de Fourier de la vitesse par rapport à $x$ :

$$
\mathbf{v}_{\mathbf{q}}(z, t)=\mathcal{A}^{-1} \int \mathrm{d}^{2} r \mathrm{e}^{\mathrm{i} \mathbf{q} \cdot \mathbf{r}} \mathbf{v}(\mathbf{r}, z, t)
$$

on trouve à $t=0$ :

$$
\begin{aligned}
& v_{z, \mathbf{q}}(z, 0)=\dot{\zeta}_{\mathbf{q}}(0) \mathrm{e}^{q z}+\frac{\zeta_{\mathbf{q}}(0) S_{2}-\dot{\zeta}_{\mathbf{q}}(0) \tau_{0}}{\left(S_{2}-S_{1}\right) \tau_{0}} \times \\
& \times\left(\mathrm{e}^{q z}-\mathrm{e}^{q z}+\sqrt{1+2 S_{1}}\right)+\frac{\zeta_{\mathbf{q}}(0) S_{1}-\dot{\zeta}_{\mathbf{q}}(0) \tau_{0}}{\left(S_{1}-S_{2}\right) \tau_{0}} \times \\
& \times\left(\mathrm{e}^{q z}-\mathrm{e}^{q z}+\sqrt{1+2 S_{2}}\right)
\end{aligned}
$$

L'état statistique du système étant décrit à l'aide des relations 17 , nous obtenons, pour la moyenne statistique du produit $v_{z, \mathbf{q}}(z, t) \zeta_{\mathbf{q}}^{*}(t)$, l'expression suivante :

$$
\begin{aligned}
& \overline{v_{z, \mathbf{q}}(z, 0) \zeta_{\mathbf{q}}^{*}(0)}= \\
&=\overline{\mid \frac{\left|\zeta_{\mathbf{q}}(0)\right|^{2}}{\tau_{0}}}\left\{\mathrm{e}^{q z}-\frac{S_{2}}{S_{2}-S_{1}} \mathrm{e}^{q z+\sqrt{1+2 S_{1}}}+\right. \\
&\left.\quad+\frac{S_{1}}{S_{2}-S_{1}} \mathrm{e}^{q z}+\sqrt{1+2 S_{2}}\right\}
\end{aligned}
$$

Cette expression n'est généralement pas nulle pour $z \neq 0$. La solution précédente implique donc que la vitesse du fluide en profondeur et le déplacement de la surface considérés au même instant, prennent des valeurs partiellement corrélées. Or ceci correspond à une hypothèse statistique tout à fait déraisonnable. On peut aussi noter que l'expression (22) dépend beaucoup des conditions d'amortissement du système : elle conduit à des valeurs numériques très faibles, quel que soit $z$, à la fois dans les conditions d'amortissement très faible $\left(y \gg 1, \tau_{0} \rightarrow \infty\right)$ ou très fort $\left(y \ll 1, S_{1} \lesssim 0,5, S_{2} \ll 1\right.$, le second membre de (22) tend vers 0 ). C'est surtout dans le domaine d'amortissement voisin de l'amortissement critique $(y \approx 1)$ que les valeurs numériques obtenues pour $v_{z, \mathbf{q}}(z, 0) \zeta_{\mathbf{q}}^{*}(0)$ sont les plus grandes $\left({ }^{8}\right)$. A titre d'exemple, la figure 1 indique la valeur numérique de cette expression en fonction de $q z$ pour une valeur particulière du paramètre $y$.

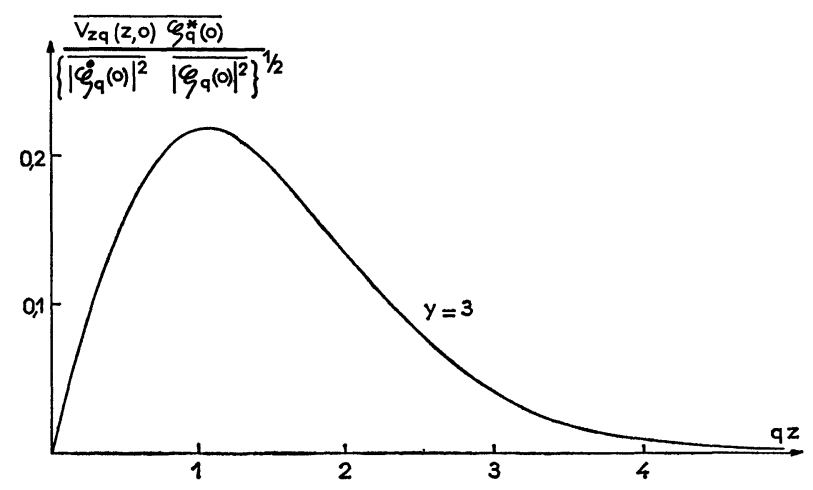

FIG. 1. - Cette figure illustre la critique apportée au calcul du $\S \mathrm{A} 4$, en donnant pour $y=3$ les valeurs numériques obtenues pour l'expression $\overline{v_{z, \mathbf{q}}(z, 0) \zeta_{\mathbf{q}}(0)} /\left\{\overline{\left|\dot{\zeta}_{\mathbf{q}}(0)\right|^{2}} \overline{\left|\zeta_{\mathbf{q}}(0)\right|^{2}}\right\}^{1 / 2}$ qui devrait être nulle quel que soit $z$.

Ce qui précède nous conduit à reprendre complètement le problème du spectre des fluctuations $\zeta_{\mathbf{q}}$. Nous imposerons cette fois comme condition que les variables $\zeta_{\mathbf{q}}(t)$ et $v_{z, \mathbf{q}}(z, t)$ considérées au même instant soient statistiquement indépendantes, quel que soit $z$. Cette condition peut être justifiée de manière rigoureuse : si l'on admet que le hamiltonien qui décrit l'état microscopique des atomes constituant le fluide est invariant par rapport au sens du temps (en l'absence de champ magnétique extérieur) la densité de probabilité est invariante par la transformation qui laisse inchangées les coordonnées des atomes et qui change leurs vitesses en leurs opposées. Par suite, toute grandeur physique qui change de signe par une \{elle transformation a une valeur moyenne nulle à l'équilibre [7] ; en particulier $\overline{v_{z, \mathbf{q}}(z, t) \zeta_{\mathbf{q}}^{*}(t)}=0$. Indiquons tout de suite pourquoi dans le calcul précédent, cette condition ne s'est pas trouvée automatiquement satisfaite en volume alors que nous l'avions imposée à la surface : la donnée de $\zeta_{\mathbf{q}}(0)$ et $\dot{\zeta}_{\mathbf{q}}(0)$ suffisent à spécifier la solution de Levich parce que c'est une solution particulière, par contre cette donnée ne suffit pas pour spécifier totalement la solution la plus générale du problème hydrodynamique. Autrement dit $\zeta_{\mathbf{q}}$ et $\dot{\zeta}_{\mathbf{q}}$ ne constituent pas un ensemble complet de variables d'état. Nous allons d'abord démontrer que la solution est complètement spécifiée, si on précise en outre la valeur à $t=0$ de $v_{z, \mathbf{q}}$ pour toutes les valeurs de $z$ (en l'astreignant toutefois à la condition $10 \mathrm{c}$ ). Ensuite, notre problème consistera à chercher la solution unique du

( ${ }^{8}$ Ceci permettra de comprendre pourquoi c'est surtout dans cette zone d'amortissement que l'on observe des différences appréciables entre le nouveau spectre calculé ci-après et l'ancien donné par la solution précédente. 
problème hydrodynamique qui correspond à l'ensemble des conditions initiales : $\zeta_{\mathbf{q}}(0)$ fixé non nul et

$$
v_{z, \mathbf{q}}(z, 0)=0
$$

quel que soit $z$. A l'aide de cette solution et de la remarque faite à la fin du $\S \mathrm{A} 2$, nous obtiendrons facilement le spectre des fluctuations dans l'hypothèse où l'état statistique $\mathrm{du}$ système est décrit à l'aide des relations :

$$
\begin{gathered}
\overline{\left|\zeta_{\mathbf{q}}(t)\right|^{2}}=\frac{k_{B} T}{\alpha q^{2} A} \\
\overline{v_{z, \mathbf{q}}(z, t) \zeta_{\mathbf{q}}^{*}(t)}=0 \text { quel que soit } z .
\end{gathered}
$$

2) RÉSOLUTION GÉNÉRALE DU PROBLÈME HYDRODYNAMIQUE. - En introduisant $\mathbf{v}_{\mathbf{q}}$ et $\mathrm{p}_{\mathbf{q}}$ les transformées de Fourier par rapport à $x$ de $\mathbf{v}$ et de $\mathrm{p}$ - $\mathrm{p}_{0}$, les équations de volume (éq. 9) peuvent se récrire sous la forme :

$$
\begin{gathered}
\mathrm{i} q v_{x, \mathbf{q}}+\frac{\partial}{\partial z} v_{z, \mathbf{q}}=0 \\
\rho \frac{\partial}{\partial t} v_{z, \mathbf{q}}=\eta\left(-q^{2} v_{z, \mathbf{q}}+\frac{\partial^{2}}{\partial z^{2}} v_{z, \mathbf{q}}\right)-\frac{\partial}{\partial z} p_{\mathbf{q}} \\
\rho \frac{\partial}{\partial t} v_{x, \mathbf{q}}=\eta\left(-q^{2} v_{x, \mathbf{q}}+\frac{\partial^{2}}{\partial z^{2}} v_{x, \mathbf{q}}\right)-\mathrm{i} q p_{\mathbf{q}} .
\end{gathered}
$$

En éliminant $v_{x, \mathbf{q}}$ et $v_{z, \mathbf{q}}$ on obtient :

$$
-q^{2} p_{\mathbf{q}}+\frac{\partial^{2}}{\partial z^{2}} p_{\mathbf{q}}=0 .
$$

La solution de cette équation qui satisfait aux conditions aux limites 10 est :

$$
p_{\mathbf{q}}=\mathrm{e}^{q z}\left\{\alpha q^{2} \zeta_{\mathbf{q}}+2 \eta\left[\frac{\partial}{\partial z} v_{z, \mathbf{q}}\right]_{z=0}\right\} \text {. }
$$

En reportant cette solution dans $(24 b)$, on obtient pour $v_{z, \mathbf{q}}$ l'équation aux dérivées partielles suivante :

$$
\begin{aligned}
\rho \frac{\partial}{\partial t} v_{z, \mathbf{q}}= & \eta\left(-q^{2} v_{z, \mathbf{q}}+\frac{\partial^{2}}{\partial z^{2}} v_{z, \mathbf{q}}\right)- \\
& -q \mathrm{e}^{q z}\left\{\alpha q^{2} \zeta_{\mathbf{q}}+2 \eta\left[\frac{\partial}{\partial z} v_{z, \mathbf{q}}\right]_{z=0}\right\}
\end{aligned}
$$

avec les conditions aux limites $10 b$ et $10 c$ qui peuvent être récrites en terme de $v_{z, q}$ seul:

$$
\begin{aligned}
& \frac{\partial^{2}}{\partial z^{2}} v_{z, \mathbf{q}}(0, t)+q^{2} v_{z, \mathbf{q}}(0, t)=0 \\
& v_{z, \mathbf{q}}(z, t) \rightarrow 0 \text { quand } z \rightarrow-\infty .
\end{aligned}
$$

Pour tenir compte des conditions initiales à satisfaire, il est pratique d'introduire les transformées de Laplace de $v_{z, \mathbf{q}}$ et $\zeta_{\mathbf{q}}$ par rapport à $t$

$$
\begin{aligned}
\tilde{v}_{z, \mathbf{q}}(z, s) & =\int_{0}^{\infty} \mathrm{d} t \mathrm{e}^{-s t} v_{z, \mathbf{q}}(z, t) \\
\tilde{\zeta}_{\mathbf{q}}(s) & =\int_{0}^{\infty} \mathrm{d} t \mathrm{e}^{-s t} \zeta_{\mathbf{q}}(t) .
\end{aligned}
$$

On utilisera la relation :

$$
\tilde{v}_{z, \mathbf{q}}(z=0, s)=-\zeta_{\mathbf{q}}(0)+s \tilde{\zeta_{\mathbf{q}}}(s)
$$

qui peut se démontrer simplement par intégration par parties.

Après avoir pris la transformée de Laplace de ses deux membres, l'équation (27) peut se récrire :

$$
\begin{gathered}
\rho s \tilde{v}_{z, \mathbf{q}}(z, s)-\eta\left[-q^{2} \tilde{v}_{z, \mathbf{q}}(z, s)+\frac{\partial^{2}}{\partial z^{2}} v_{z, \mathbf{q}}(z, s)\right]+ \\
+q \mathrm{e}^{q z}\left\{\alpha q^{2} \zeta_{\mathbf{q}}(s)+2 \eta\left[\frac{\partial}{\partial z} \tilde{v}_{z, \mathbf{q}}(z, s)\right]_{z=0}\right\}= \\
=\rho v_{z, \mathbf{q}}(z, 0) .
\end{gathered}
$$

Nous pouvons transformer cette équation en une équation différentielle d'ordre 3 par rapport à $z$ en multipliant ses deux membres par $\mathrm{e}^{-q z}$ et en les différentiant une fois par rapport à $z$. On obtient :

$$
\frac{\mathrm{d}}{\mathrm{d} z}\left[\mathrm{e}^{-q z}\left(a u^{\prime \prime}+b u\right)\right]=\frac{\mathrm{d}}{\mathrm{d} z}\left[\mathrm{e}^{-q z} \varphi(z)\right]
$$

ou

$$
a u^{\prime \prime \prime}-a q u^{\prime \prime}+b u^{\prime}-b q u=\psi(z)
$$

avec

$$
\begin{gathered}
a=-\eta, \quad b=+s \rho+\eta q^{2} \\
\varphi(z)=\rho v_{z, \mathbf{q}}(z, t=0) \\
\psi(z)=\frac{\mathrm{d}}{\mathrm{d} z} \varphi(z)-q \varphi(z) \\
u(z)=\tilde{v}_{z . \mathbf{q}}(z, s)
\end{gathered}
$$

$s$ est considéré comme un paramètre.

On cherche la solution de 33 qui satisfait les 3 conditions :

$$
\begin{gathered}
a u^{\prime \prime}(0)+\gamma u^{\prime}(0)+(b+\beta) u(0)=\delta \\
u^{\prime \prime}(0)+q^{2} u(0)=0 \\
u(z) \rightarrow 0 \text { quand } z \rightarrow-\infty .
\end{gathered}
$$

La première a été obtenue en récrivant l'équation 31 pour $z=0$, compte tenu de 30 et en introduisant les notations suivantes :

$\beta=\frac{\alpha q^{3}}{s}, \gamma=2 \eta q, \delta=\frac{-\alpha q^{3} \zeta_{\mathbf{q}}(0)}{s}+\rho \dot{\zeta}_{\mathbf{q}}(0)$.

Les conditions $35 b$ et $35 c$ sont équivalentes à $28 b$ et $28 c$.

Le problème mathématique auquel on est ramené est finalement très simple. Sa solution est unique. On peut s'en assurer en notant que si 2 fonctions $u_{1}$ et $u_{2}$ satisfont à la fois les équations 33 et 35 , leur différence est solution de 33 et 35 correspondant au cas particulier où $\delta=0$ et $\psi(z) \equiv 0$, et il est facile de 
vérifier que ce problème n'admet qu'une solution identiquement nulle $\left(u_{1}-u_{2} \equiv 0\right)$.

Nous avons donc montré que $\zeta_{\mathbf{q}}(0)$ et $v_{z, \mathbf{q}}(z, 0)$ forment un ensemble complet de variables d'état. Nous pouvons maintenant utiliser la remarque mentionnée à la fin du $\S \mathrm{A} 2$ et nous limiter à calculer explicitement la solution du problème hydrodynamique satisfaisant à la condition initiale $v_{z, \mathbf{q}}(z, t) \equiv 0$ et à une valeur initiale fixée de $\zeta_{\mathbf{q}}$. Ceci revient à chercher la fonction qui satisfait 33 et 35 dans le cas où $\psi(z) \equiv 0$, soit :

$$
\tilde{v}_{z, \mathbf{q}}(z, s)=f(s) \mathrm{e}^{q z}+g(s) \mathrm{e}^{m(s) z}
$$

avec

$$
m(s)=q \sqrt[+]{1+2 s \tau_{0}} .
$$

En explicitant $f$ et $g$, on obtient :

$$
\tilde{v}_{z, \mathbf{q}}(z=0, s)=-\frac{y \zeta_{\mathbf{q}}(0)}{\left[y+(1+S)^{2}-\sqrt[+]{1+2 S}\right]_{S=s \tau_{0}}}
$$

ce qui conduit à :

$$
\begin{aligned}
\tilde{\zeta}_{\mathbf{q}}(s) & =\frac{\tilde{v}_{z, \mathbf{q}}(z=0, s)}{s}+\frac{\underline{\zeta_{\mathbf{q}}}(0)}{s} \\
& =\left\{\frac{(1+S)^{2}-\sqrt[+]{1+2 S}}{s\left[y+(1+S)^{2}-\sqrt[+]{1+2 S}\right]}\right\}_{S=s \tau_{0}} \zeta_{\mathbf{q}}(0) .
\end{aligned}
$$

Les paramètres $\tau_{0}$ et $y$ ont été définis précédemment (éq. 11). On voit que la transformée de Laplace inverse de $\tilde{\zeta}_{\mathbf{q}}(s)$ n'est pas simple; avec ce choix de conditions initiales $\tilde{\zeta}_{\mathbf{q}}(t)$ n'est pas de caractère exponentiel. On peut noter aussi que $v_{z, \mathbf{q}}$ obtenu par transformation de Laplace inverse de (37 a) n'est pas le produit d'une fonction de $t$ seul par une fonction de $z$ seul. Comme nous l'avions annoncé, la solution construite dans ce paragraphe n'entre donc pas dans le type des solutions particulières calculées par Levich.

3) Calcul du spectre des fluctuations. - En utilisant le procédé décrit au § A 2, et le calcul précédent, nous pouvons maintenant construire non pas exactement la fonction de corrélation des fluctuations de surface, mais sa transformée de Laplace :

$$
\begin{aligned}
& \overline{\tilde{\zeta}_{\mathbf{q}}(s) \zeta_{\mathbf{q}}^{*}(0)}= \\
& \quad=\left\{\frac{(1+S)^{2}-\sqrt[+]{1+2 S}}{\left|y+(1+S)^{2}-\sqrt[+]{1+2 S}\right| s}\right\}_{S=s \tau_{0}} \mid \overline{\left.\zeta_{\mathbf{q}}(0)\right|^{2}}
\end{aligned}
$$

dans l'hypothèse où l'état statistique du fluide à l'équilibre satisfait à la condition

$$
\overline{v_{z, \mathbf{q}}(z, t) \zeta_{\mathbf{q}}^{*}(t)}=0 \text { quel que soit } z \text {. }
$$

On remarquera qu'avec les définitions utilisées (éq. 4 et $29 \mathrm{~b}$ ), la relation qui lie le spectre des fluctuations à l'expression calculée ci-dessus s'écrit :

$$
\begin{aligned}
\left\{\overline{\tilde{\zeta}_{\mathbf{q}}(s) \zeta_{\mathbf{q}}^{*}(0)}\right. & + \text { c. c. }\}_{s=-\mathrm{i} \omega}= \\
& =\int_{-\infty}^{+\infty} G_{\mathbf{q}}(\tau) \mathrm{e}^{\mathrm{i} \omega \tau} \mathrm{d} \tau=2 \pi P_{\mathbf{q}}(\omega) .
\end{aligned}
$$

D'où le spectre :

$$
\begin{gathered}
P_{\mathbf{q}}(\omega)=\frac{1}{\pi} \operatorname{Re}\left\{\overline{\zeta_{\mathbf{q}}(s) \zeta_{\mathbf{q}}^{*}(0)}\right\}_{s=-\mathrm{i} \omega}= \\
=-\frac{1}{\pi \omega} y\left\{\frac{\operatorname{Im}\left[y+(1+S)^{2}-\sqrt[+]{1+2 S}\right]}{\mid y+\frac{(1+S)^{2}-\left.\sqrt{1+2 S}\right|^{2}}{\mid y=-\mathrm{i} \omega \tau_{0}}} \times\right. \\
\times \sqrt{\left|\zeta_{\mathbf{q}}(0)\right|^{2}} .
\end{gathered}
$$

En substituant la valeur de $\overline{\left.\zeta_{\mathbf{q}}(0)\right|^{2}}$ (équation $17 a$ ) et en tenant compte de ce que $P_{\mathbf{q}}(\omega)$ est une fonction paire de $\omega$ :

$P_{\mathrm{q}}(\omega)=\frac{q}{\rho} \tau_{0}^{2}\left\{\frac{\operatorname{Im}[2 S-\sqrt[+]{1+2 S}]}{\left|y+(1+S)^{2}-\sqrt[+]{1+2 S}\right|^{2}}\right\}_{S=\mathrm{i} \omega \tau_{0}} \frac{k_{B} T}{\omega \pi}$.

On reconnaît au dénominateur de cette expression l'équation de dispersion du système (éq. $15 a$ ). Si celle-ci possède 2 racines imaginaires conjuguées $S_{1,2}=S_{r} \pm$ i $S_{i}$, le spectre présente 2 maximums prononcés au voisinage de $\omega= \pm S_{i}$. On peut facilement vérifier que ceci est vrai aussi pour le spectre donné par l'expression (20). Une comparaison numérique détaillée (voir $\S \mathrm{C}$ ) permet de montrer que les 2 types de spectres (éq. 20 et 43 ) sont pratiquement identiques dans les conditions d'amortissement fort ou faible $(y \gg 1$ ou $y \ll 1)$. Par contre, il existe aussi une petite zone d'amortissement proche du point d'amortissement critique pour laquelle la différence entre les deux n'est pas négligeable $(0,3 \lesssim y \lesssim 3)$.

4) VÉRIFICATION DU THÉORÈME DE FLUCTUATIONDisSIPATION. - Les fluctuations d'une grandeur physique caractéristiques d'un système à l'équilibre peuvent être reliées au phénomène de dissipation associé à la réponse de ce système lorsqu'il est soumis à une force extérieure [8]. Dans le cas présent, envisageons la surface libre soumise à une action extérieure capable de la déformer. Ceci correspond à l'apparition dans l'énergie de la surface d'un terme de perturbation du type $-\int \mathrm{d}^{2} r \zeta(\mathbf{r}) \Pi(\mathbf{r}, t)$ où $\Pi$ traduisant l'action d'une source extérieure est une pression dépendant du temps.

La forme la plus générale du déplacement de la surface $\zeta(r, t)$ en réponse à la perturbation extérieure. en se limitant aux effets linéaires, peut s'écrire :

$\zeta(\mathbf{r}, t)=\int_{S} \mathrm{~d}^{2} r^{\prime} \int_{0}^{\infty} \chi\left(\mathbf{r}-\mathbf{r}^{\prime}, \tau\right) \Pi\left(\mathbf{r}^{\prime}, t-\tau\right) \mathrm{d}^{2} r^{\prime} \mathrm{d} \tau$

ou encore pour un mode de surface donné :

$$
\zeta_{\mathbf{q}}(t)=\int_{0}^{\infty} \chi_{\mathbf{q}}(\tau) \Pi_{\mathbf{q}}(t-\tau) \mathrm{d} \tau
$$

Si $\Pi_{\mathbf{q}}(t)$ varie comme $\Pi_{\mathbf{q}}^{\omega} \mathrm{e}^{-\mathrm{i} \omega t}$, il en est de même de $\zeta_{\mathbf{q}}(t)$ et l'on a

$$
\widetilde{\widetilde{\zeta}}_{\mathbf{q}}(\omega)=\approx_{\chi_{\mathbf{q}}}(\omega) \Pi_{\mathbf{q}}^{\omega}
$$


avec

$$
\tilde{\tilde{\chi}}_{\mathbf{q}}(\omega)=\int_{0}^{\infty} \mathrm{d} \tau \chi_{\mathbf{q}}(\tau) \mathrm{e}^{\mathrm{i} \omega \tau}
$$

Nous nous proposons de calculer explicitement la fonction de réponse $\widetilde{\tilde{\chi}}_{\mathbf{q}}(\omega)$ et de vérifier que le spectre des fluctuations thermiques de la grandeur $\zeta_{\mathbf{q}}$ calculé précédemment, lui est bien relié comme le prévoit le théorème de fluctuation-dissipation. D'après ce théorème, dans la limite classique $\hbar \omega \ll k_{B} T$, on doit vérifier la relation :

$$
P_{\mathbf{q}}(\omega)=\frac{k_{B} T}{\pi \omega} \operatorname{Im} \widetilde{\tilde{\chi}}_{\mathbf{q}}(\omega)
$$

Le calcul de $\tilde{\chi}_{\mathbf{q}}(\omega)$ revient à chercher la solution du problème hydrodynamique satisfaisant toujours aux mêmes équations de volume (éq. 10), mais à des conditions aux limites différentes: dans l'équation (10 a) exprimant l'équilibre des contraintes normales à la surface s'introduit le terme dû à la pression extérieure :

$-p+2 \eta \frac{\partial v_{z}}{\partial z}-\alpha \frac{\partial^{2} \zeta}{\partial x^{2}}=-\Pi-p_{0}$ pour $z=0$.

Les conditions $10 b$ et $10 c$ demeurent évidemment inchangées.

On peut résoudre ce nouveau problème en suivant pas à pas le même procédé que précédemment pour le problème aux conditions initiales, aussi nous indiquerons seulement les étapes essentielles.

Après transformation de Fourier par rapport à $x$, on obtient pour la pression la solution suivante:

$$
p_{\mathbf{q}}=\mathrm{e}^{q z}\left\{\alpha q^{2} \zeta_{\mathbf{q}}+2 \eta\left[\frac{\partial}{\partial z} v_{z, \mathbf{q}}\right]_{z=0}+\Pi_{\mathbf{q}}\right\} .
$$

On substitue ce résultat dans $(24 b)$ et on prend la transformée de Fourier de ses 2 membres en introduisant :

$$
\tilde{\tilde{v}}_{z, \mathbf{q}}(z, \omega)=(2 \pi)^{-1} \int_{-\infty}^{+\infty} \mathrm{d} t v_{z, \mathbf{q}}(z, t) \mathrm{e}^{\mathrm{i} \omega t}
$$

et

$$
\begin{gathered}
\tilde{\Pi}_{\mathbf{q}}^{\omega}=\Pi_{\mathbf{q}}^{\omega} \delta(\omega) \\
-\mathrm{i} \omega \rho \tilde{\tilde{v}}_{z, \mathbf{q}}-\eta\left\{-q^{2} \tilde{\tilde{v}}_{z, \mathbf{q}}+\frac{\partial^{2}}{\partial z^{2}} \tilde{\tilde{v}}_{z, \mathbf{q}}\right\}+ \\
+q \mathrm{e}^{q z}\left\{\left[\alpha q^{2} \frac{\tilde{\tilde{v}}_{z, \mathbf{q}}}{-\mathrm{i} \omega}+2 \eta \frac{\partial}{\partial z} \tilde{\tilde{v}}_{z, \mathbf{q}}\right]_{z=0}+\tilde{\tilde{\Pi}}_{\mathbf{q}}^{\omega}\right\}=0
\end{gathered}
$$

La solution cherchée doit satisfaire les conditions aux limites

$$
\begin{gathered}
\left\{\frac{\partial^{2}}{\partial z^{2}} \tilde{v}_{z, \mathbf{q}}+q^{2} \tilde{\tilde{v}}_{z, \mathbf{q}}\right\}_{z=0}=0 \\
\tilde{\tilde{v}}_{z, \mathbf{q}}(z, \omega) \rightarrow 0 \text { quand } z \rightarrow-\infty .
\end{gathered}
$$

A ce stade, le problème est exactement ramené au précédent : chercher la solution de 33 satisfaisant les 3 conditions 35 , avec maintenant pour notations :

$$
\begin{aligned}
& u=\tilde{\tilde{v}}_{z, \mathbf{q}}(z, \omega) \\
& a=-\eta, \quad b=-\mathrm{i} \omega \rho+\eta q^{2} \\
& \beta=-\frac{\alpha q^{3}}{\mathrm{i} \omega}, \quad \gamma=2 \eta q, \quad \delta=q \tilde{\tilde{\Pi}}_{\mathbf{q}}^{\omega} .
\end{aligned}
$$

La solution est donnée par l'expression :

$$
\begin{gathered}
\approx_{z, \mathbf{q}}(z, \omega)= \\
=\left\{\left(1-\mathrm{i} \omega \tau_{0}\right) \mathrm{e}^{q z}-\mathrm{e}^{q z} \sqrt[+]{1-2 \mathrm{i} \omega \tau 0}\right\} \tilde{\zeta}_{\mathbf{q}}(\omega) \tau_{0}^{-1}
\end{gathered}
$$

avec

$$
\begin{aligned}
\tilde{\zeta}_{\mathbf{q}}(\omega) & =\left[\frac{\tilde{\tilde{v}}_{z, \mathbf{q}}(z, \omega)}{-\mathrm{i} \omega}\right]_{z=0}= \\
& =-\tau_{0}^{2} \frac{q}{\rho} \frac{\tilde{\tilde{\Pi}}_{\mathbf{q}}^{\omega}}{\left[y+(1+S)^{2}-\sqrt[+]{1+2 S}\right]_{S=-\mathrm{i} \omega \tau_{0}}} .
\end{aligned}
$$

On obtient ainsi pour la fonction de réponse :

$$
\begin{gathered}
\operatorname{Im} \tilde{\tilde{\chi}}_{\mathbf{q}}(\omega)= \\
=-\tau_{0}^{2} \frac{q}{\rho}\left\{\frac{\operatorname{Im}(2 S-\sqrt[+]{1+2 S)}}{\left[y+(1+S)^{2}-\sqrt[+]{1+2 S}\right]^{2}}\right\}_{S=-\mathrm{i} \omega \tau_{0}} .
\end{gathered}
$$

En rapprochant ce résultat et le précédent relatif à $P_{\mathbf{q}}(\omega)$ (éq. 43 ), on vérifie bien le théorème de fluctuation-dissipation (éq. 48).

5) Remarque SUR LA FONCTION DE RÉPONSE DU SYSTÈME A UNE PRESSION SINUSOÏDALE EXTÉRIEURE. Nous avons obtenu pour fonction de réponse du système

$$
\tilde{\chi}_{\mathbf{q}}(\omega)=\frac{-\tau_{0}^{2} q / \rho}{y+\left(1-\mathrm{i} \omega \tau_{0}\right)^{2}-\sqrt[+]{1-2 \mathrm{i} \omega \tau_{0}}} .
$$

On peut vérifier que si l'on donne des valeurs complexes à la variable $\omega$ ci-dessus, on obtient bien une fonction analytique dont toutes les singularités sont situées dans le même demi-plan $(\operatorname{Im} \omega<0)$; propriété générale d'un système physique causal [8]. Parmi ces singularités, il y a les 2 pôles qui correspondent aux 2 racines de l'équation de dispersion ayant un sens physique (relations 15), c'est-à-dire aux 2 fréquences propres $\mathrm{du}$ système ; il y a aussi un point de branchement (situé sur l'axe $\omega$ imaginaire pour $\omega=-\mathrm{i} / 2 \tau_{0}$ ).

Notons que le spectre approché (éq. 20) obtenu lors du calcul antérieur (cf. $\S \mathrm{A} \mathrm{4}$ ) vérifie le théorème de fluctuation-dissipation à condition de prendre pour expression approchée de la fonction de réponse

$$
\tilde{\tilde{\chi}}_{\mathbf{q}}(\omega)=\frac{-S_{1} S_{2} / \alpha q^{2}}{-\omega^{2} \tau_{0}^{2}-\left(S_{1}+S_{2}\right) i \omega \tau_{0}+S_{1} S_{2}}
$$

à la place de l'expression exacte (58). La fonction analytique correspondant à (59) a les 2 mêmes pôles que (58), elle en diffère seulement par l'absence du 
point de branchement. Cette dernière singularité peut être associée au phénomène de dispersion qui accompagne la propagation du mouvement de la surface vers les couches fluides sous-jacentes. En effet, il ressort du calcul précédent que si l'on produit un déplacement à la surface $\zeta_{\mathbf{q}}(t)$, le déplacement vertical du fluide à la cote $z, u_{q}(z, t)$, qui en résulte est de la forme :

$$
u_{\mathbf{q}}(z, t)=\int_{0}^{\infty} \mathrm{d} \tau K(z, \tau) \zeta_{\mathbf{q}}(t-\tau)
$$

avec

$$
\begin{aligned}
& \tilde{\widetilde{K}}(z, \omega)=(2 \pi)^{-1} \int_{0}^{\infty} \mathrm{d} \tau K(z, \tau) \mathrm{e}^{\mathrm{i} \omega \tau} \\
& =\mathrm{e}^{q z}+\frac{\mathrm{i}}{\omega \tau_{0}}\left(\mathrm{e}^{q z} \sqrt[+]{1-2 \mathrm{i} \omega \tau_{0}}-\mathrm{e}^{q z}\right) .
\end{aligned}
$$

Le premier terme est indépendant de $\omega$ : il traduit une excitation instantanée, tandis que le second est caractérisé par un certain retard; le terme qui lui correspond dans $K(z, \tau)$ n'est pas fonction exponentielle du temps, mais un ordre de grandeur du retard est donné par $\tau_{0}$.

Les 2 termes ci-dessus correspondent à l'établissement des 2 modes de volume qui sont associés à un mode de surface donné : le premier correspond à un écoulement potentiel (rot $\mathbf{v}=0$ ), le second à un écoulement rotationnel (rot $\mathbf{v} \neq 0$ ) $\left({ }^{9}\right)$. Dans le cas d'un fluide idéal $(\eta=0)$ où le premier mode existe seul, le mouvement de la surface fait apparaître une modification de pression dans le fluide qui se transmet quasi instantanément aux couches sous-jacentes (le fluide est supposé incompressible) : le fluide se met en mouvement d'un seul bloc. Le mode rotationnel s'établit de manière différente : la déformation de la surface crée des lignes de tourbillon (d'axe parallèle à $\mathrm{Oy}$ ) qui diffusent dans le fluide sous-jacent avec dissipation d'énergie; le mouvement s'établit avec un retard de l'ordre de $\tau_{0}$.

En conclusion, les phénomènes de dissipation qui entrent en jeu dans le volume du fluide situé juste au-dessous de la surface libre peuvent influencer de manière appréciable le spectre des ondes de surface excitées thermiquement.

C) Comparaison entre spectres. - 1) CoMParaison DES SPECTRES THÉORIQUES ENTRE EUX. - La figure 2 permet de comparer, pour différentes valeurs de $y$ $(0,145,0,3$ et 3$)$, le spectre des fluctuations thermiques de la surface libre (courbe $a$, correspondant à l'équation 43), et le spectre des fluctuations de la coordonnée d'un oscillateur harmonique (courbe $b$, éq. 20). A l'extérieur du domaine d'amortissement envisagé ici $(0,145 \lesssim y \lesssim 3)$, les différences sont si faibles qu'elles

(9) La définition et les propriétés de ces 2 types d'écoulement se trouvent par exemple dans la référence [6].
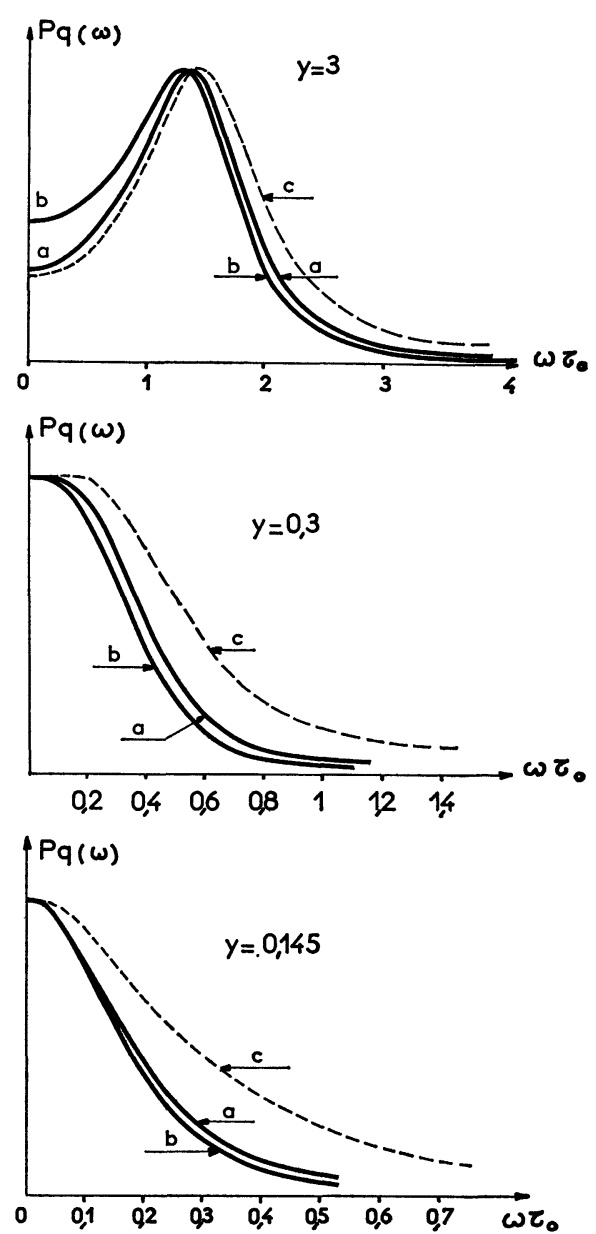

FIG. 2. - Spectre théorique des fluctuations thermiques de la surface libre (courbe $a$ ), spectre des fluctuations de la coordonnée d'un oscillateur harmonique (courbe $b$ ), et spectre correspondant à la superposition de 2 courbes de Lorentz (courbe $c$ ), construits pour la même valeur du paramètre $y=\alpha \rho / 4 \eta^{2} q$, en unités réduites de pulsation $\left(\tau_{0}=\rho / 2 \eta q^{2}\right)$.

ne pourraient être représentées de cette manière. On remarque que les deux spectres présentent le maximum d'écart dans le cas périodique très amorti $y=3$, au voisinage de la fréquence 0 , c'est-à-dire dans une zone où il est difficile de rendre les mesures expérimentales précises. Pour les régimes encore plus amortis $(y=0,3$ et $y=0,145)$ où le spectre est centré à la fréquence 0 , on voit que les 2 spectres ont des formes très semblables (en particulier même décroissance en $1 / \omega^{4}$ sur l'aile). Pour chaque valeur de $y$, on a aussi tracé en pointillé la courbe qui correspond à la somme de 2 courbes de Lorentz centrées à $\omega= \pm S_{i} \tau_{0}^{-1}$ et de largeur $\gamma=S_{r} \tau_{0}^{-1}$ (courbe $c$ ). Notons qu'elle s'écarte plus dans l'ensemble des courbes $a$ et $b$ que ces deux dernières entre elles.

2) COMPARAISON DES SPECTRES THÉORIQUES ET EXPÉRIMENTAUX. - Au cours d'une première étude expérimentale détaillée [5], aucun désaccord systématique n'a pu être observé entre les spectres expérimentaux et le spectre des fluctuations thermiques d'un oscillateur harmonique. Ceci n'est pas très surprenant étant 
donné ce qui précède : la précision expérimentale dont on disposait alors, n'était pas suffisante pour déceler la différence. De multiples améliorations viennent de nous permettre d'étudier récemment le spectre sur un nouveau montage avec une précision bien supérieure, surtout aux fréquences voisines de 0 . La figure 3

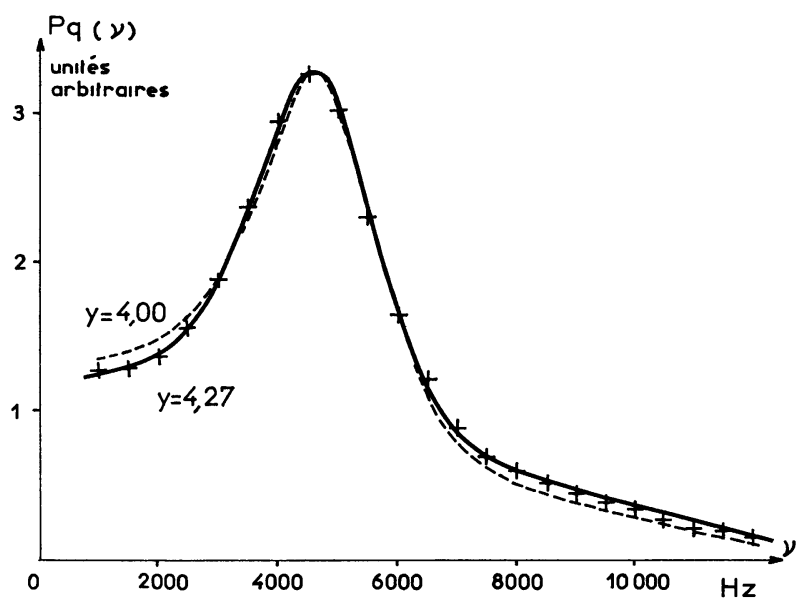

FIG. 3. - Etude spectrale de la lumière diffusée par l'interface liquide-vapeur de $\mathrm{CO}_{2}$ à $t=30,438{ }^{\circ} \mathrm{C}$ pour $q=3325 \mathrm{~cm}^{-1}$. Les points sont expérimentaux, les 2 courbes sont théoriques : celle en trait plein correspond au spectre théorique des fluctuaions de surface (éq. 62 et 64 ), celle en pointillé au spectre des fluctuations de la coordonnée d'un oscillateur harmonique (éq. 62,20 et 67 ).

représente les points expérimentaux avec, en trait plein, la courbe théorique correspondant aux fluctuations de surface et, en trait pointillé, la courbe théorique donnant le spectre des fluctuations d'un oscillateur harmonique. Chacune de ces courbes théoriques a été choisie de manière à rendre minimum l'écart quadratique moyen entre les points expérimentaux et la courbe (ceci explique qu'elles ne corresponcent pas exactement à la même valeur du paramètre $y$ ). C'est la première courbe théorique qui donne le meilleur accord avec les points expérimentaux : l'écart carré moyen des points à la courbe est 4 fois plus faible pour elle que pour l'autre ; il est intéressant de $r \in$ marquer que l'accord est particulièrement bon aux basses fréquences, là où les 2 courbes théoriques diffèrent le plus. Nous espérons pouvoir améliorer encore un peu la précision avec laquelle est vérifiée la forme spectrale. Le spectre présenté sur la figure 2 n'est pas comparé exactement au spectre $P_{\mathbf{q}}(\omega)$ mais à l'expression :

$$
P_{\mathbf{q}}(\omega)+\lambda \frac{\int \mathrm{d} \omega^{\prime} P_{q}\left(\omega^{\prime}\right) P_{q}\left(\omega-\omega^{\prime}\right)}{\int \mathrm{d} \omega^{\prime} P_{\mathbf{q}}\left(\omega^{\prime}\right)}
$$

$\lambda$ étant un paramètre ajustable qui représente le rapport entre les rendements homodyne et hétéroỏyne [9] au cours de l'expérience. Pour les 2 courbes théoriques, le meilleur accord est obtenu avec des valeurs de $\lambda$ légèrement différentes, voisines de 0,3. A l'heure actuelle, la valeur de $\lambda$ est difficilement contrôlable, mais si l'on parvient dans la suite à modifier la détec- tion de manière à rendre $\lambda$ soit très petit, soit très grand devant 1 , il est certain que la vérification deviendra plus précise.

Remarque. - Les points expérimentaux présentés sur la figure 3 ont été obtenus pour la diffusion de la lumière non pas par la surface d'un liquide simple, mais par l'interface liquide-vapeur de $\mathrm{CO}_{2}$ à $0,7^{\circ}$ environ de son point critique. Les 2 phases fluides en contact ont des densités $\rho$ et $\rho^{\prime}$ et des viscosités $\eta$ et $\eta^{\prime}$ différentes, qui satisfont avec une très bonne approximation la relation

$$
\frac{\rho}{\rho^{\prime}}=\frac{\eta}{\eta^{\prime}}
$$

La généralisation à ce dernier cas des calculs faits au $\S$ B ne pose pas de difficultés; nous précisons l'expression algébrique c'u résultat car c'est elle qui a été utilisée pour tracer la courbe en trait plein de la figure 2 :

$$
P_{\mathbf{q}}(\omega)=\frac{k_{B} T}{\pi \omega} \cdot \frac{q}{\rho+\rho^{\prime}} \tau_{0}^{2} \operatorname{Im}\left\{\mathcal{F}^{-1}\left(\mathrm{i} \omega \tau_{0}\right)\right\}
$$

avec

$$
\begin{gathered}
\tau_{0}=\frac{\rho+\rho^{\prime}}{2\left(\eta+\eta^{\prime}\right) q^{2}}, \quad y=\frac{\alpha\left(\rho+\rho^{\prime}\right)}{4\left(\eta+\eta^{\prime}\right)^{2} q} \\
\mathcal{F}(S)=y+\frac{2 \rho \rho^{\prime}}{\left(\rho+\rho^{\prime}\right)^{2}} S^{+} \sqrt{1+2 S}[1+\sqrt[+]{1+2 S}]+ \\
+\frac{\left(\rho-\rho^{\prime}\right)^{2}}{\left(\rho+\rho^{\prime}\right)^{2}}\left[(1+S)^{2}-\sqrt[+]{1+2 S}\right]
\end{gathered}
$$

La courbe théorique tracée en pointillé correspond à $P_{\mathbf{q}}(\omega)$ donné par l'équation $20, S_{1}$ et $S_{2}$ étant les 2 racines à partie réelle positive de l'équation de dispersion :

$$
\mathscr{F}(S)=0 \text {. }
$$

Conclusion. - Nous avons appliqué la théorie thermodynamique des fluctuations au calcul du spectre des fluctuations thermiques de la surface libre d'un liquide simple. Nous avons montré que la solution rigoureuse du problème faisait intervenir l'état statistique de la surface et aussi du fluide sous-jacent. En calculant séparément la fonction de réponse de la surface à une force de pression extérieure, nous avons $\mathrm{pu}$ vérifier que le spectre obtenu satisfaisait bien au théorème de fluctuation-dissipation. Il présente des différences non négligeables vis-à-vis du spectre des fluctuations thermiques d'un oscillateur harmonique. Par ces différences se manifestent les phénomènes de retard et de dissipation qui accompagnent la propagation du mouvement dans un fluide visqueux, de la surface vers les couches fluides situées au-dessous. Nous avons montré un spectre expérimental où les différences prévues apparaissent de manière significative.

Remerciements. - L'un de nous (M. A. B.) est reconnaissant à Claude Bouchiat pour les discussions fructueuses que nous avons eues au sujet de ce travail. 


\section{Bibliographie}

[1] FABELINSKII (I. L.), Molecular Scattering of Light, Plenum Press, N. Y. 1968, p. 303.

[2] Bouchiat (M. A.), Meunier (J.) et Brossel (J.), C. $R$. Acad. Sci. Paris, 1968, 266, 255 et 301 ; KATYL (R. H.) and IngARD (U.), Phys. Rev. Letters, 1968, 20, 248 ; Papoular (M.), J. Physique, 1968, 29, 81.

[3] Landau (L. D.) et Placzek (G.), Physik z. Sowjetunion, 1934, 5, 172

[4] Mountain (R. D.), Rev. Mod. Phys., 1966, 38, 205.

[5] Meunier (J.), Cruchon (D.) et Bouchiat (M. A.), C. R. Acad. Sci. Paris, 1969, 268, 92 et 422 .

[6] Levich (V. G.), Physicochemical Hydrodynamics, chap. XI.
[7] Nous sommes reconnaissants à BouChIAT (Cl.) pour cette remarque qui permet de justifier de manière rigoureuse les conditions initiales du problème.

[8] Landau (L. D.), Physique statistique, Moscou, 1967, chap. XII.

[9] Bentede (G. B.), Polarisation, Matière et Rayonnement, édité par la Société Française de Physique (Presses Universitaires de France, Paris, France, 1969), p. 49 ou Cummins (H. Z.) and SwinNEY (H. L.), Progress in Optics, Volume VIII, edited by Wolf (E.) (North Holland Publ. Co., Amsterdam, 1970), p. 135. 